Article

\title{
Investigating Algorithmic Misconceptions in a Media Context: Source of a New Digital Divide?
}

\author{
Brahim Zarouali ${ }^{1, *}$, Natali Helberger ${ }^{2}$ and Claes H. de Vreese ${ }^{1}$ \\ ${ }^{1}$ Amsterdam School of Communication Research, University of Amsterdam, The Netherlands; \\ E-Mails: b.zarouali@uva.nl (B.Z.), c.h.devreese@uva.nl (C.H.d.V.) \\ 2 Institute for Information Law, University of Amsterdam, The Netherlands; E-Mail: n.helberger@uva.nl \\ * Corresponding author
}

Submitted: 29 January 2021 | Accepted: 19 April 2021 | Published: 18 November 2021

\begin{abstract}
Algorithms are widely used in our data-driven media landscape. Many misconceptions have arisen about how these algorithms work and what they can do. In this study, we conducted a large representative survey $(N=2,106)$ in the Netherlands to explore algorithmic misconceptions. Results showed that a significant part of the general population holds (multiple) misconceptions about algorithms in the media. We found that erroneous beliefs about algorithms are more common among (1) older people (vs. younger people), (2) lower-educated people (vs. higher-educated), and (3) women (vs. men). In addition, it was found that people who had no specific sources to inform themselves about algorithms, and those relying on their friends/family for information, were more likely to have algorithmic misconceptions. Conversely, media channels, school, and having one's own (online) experiences were found to be sources associated with having fewer algorithmic misconceptions. Theoretical implications are formulated in the context of algorithmic awareness and the digital divide. Finally, societal implications are discussed, such as the need for algorithmic literacy initiatives.
\end{abstract}

\section{Keywords}

algorithms; algorithmic awareness; digital divide; misconceptions; technology

\section{Issue}

This article is part of the issue "Algorithmic Systems in the Digital Society" edited by Sanne Kruikemeier (University of Amsterdam, The Netherlands), Sophie Boerman (University of Amsterdam, The Netherlands), and Nadine Bol (Tilburg University, The Netherlands).

(C) 2021 by the authors; licensee Cogitatio (Lisbon, Portugal). This article is licensed under a Creative Commons Attribution 4.0 International License (CC BY).

\section{Introduction}

In our data-driven media landscape, algorithms play an increasingly important role in how online users use, navigate, and consume online information and communication (Beer, 2017; Lee, 2018; Ricci, 2015). For instance, recommendation algorithms allow online platforms and legacy media alike to make personalized recommendations based on people's profiles; content moderation algorithms are used to determine the ranking of the contents that are being shown to us; automated filtering algorithms allow us to detect instances of misinformation, harmful, or unlawful content; etc. Given their widespread use and impact on people's media and infor- mation consumption, having a proper sense of what algorithms are and are capable of doing is a necessary condition for digital citizenship. However, recent studies have indicated that a significant part of the population has limited knowledge about the algorithms used in online platforms (e.g., Facebook, Google, etc.), as well as misconceptions about how they work (Cotter \& Reisdorf, 2020; e.g., Eslami et al., 2015; Rader \& Gray, 2015).

Misconceptions refer to incorrect ideas formed as a result of unfounded concerns and erroneous beliefs. These ideas may be due to the increased hype about the promises of algorithms and machine learning, which has fueled a variety of false assumptions (de Saint Laurent, 2018). These misconceptions can raise some 
serious issues, such as a highly polluted public debate about algorithms (with many loud voices that contribute to a dystopian view of the future) and missing out on the full potential of algorithms for societal good (de Saint Laurent, 2018; Elish \& boyd, 2018; First, 2018; Frank et al., 2017). Maybe even more seriously, misconceptions about the workings and consequences of algorithms can contribute to major societal problems, such as the spread of misinformation and deep fakes, data-driven manipulation and re-enforcing stereotypes, and inequalities and discrimination (Eubanks, 2017; Mohamed et al., 2020).

As an integral part of education, misconceptions must be addressed to avoid anxiety, fatalism, and distress about technological developments. Critical to this effort is knowing the extent to which these misconceptions have infiltrated our society, as well as from where they originate. Drawing on the theoretical tenets of algorithmic awareness, we present findings from a large representative survey $(N=2,106)$ in the Netherlands in which we explore the prevalence of various misconceptions about algorithms and their distribution among demographic groups, as well as mapping out the main information sources related to these misconceptions. In a concluding discussion section, we address the societal implications of the findings, as well as the theoretical contributions to the literature of algorithmic awareness, algorithmic accountability, and the digital divide. Finally, we address how to overcome these misconceptions and empower people to become informed citizens in the age of information technologies.

\section{Literature Review}

\subsection{Algorithmic Awareness}

Algorithms can be described as codified procedures for transforming vast amounts of input data into the desired output, based on specified calculations (Gillespie, 2014). From a technical perspective, algorithms are very complicated entities and are part of a larger and equally complex socio-technical infrastructure (Kitchin, 2017). In addition, they serve as an important competitive advantage for many companies (e.g., big tech platforms), which explains why such companies are very reluctant to expose their algorithmic codes to the outside world (Pasquale, 2015). Given this technical complexity and increased lack of transparency, it is very hard to be exactly aware of what algorithms are doing (Cotter \& Reisdorf, 2020). Adding to the lack of transparency is the fact that many algorithmic applications strive to provide a seamless user experience, optimizing for invisibility and normalization over time. However, despite these constraints, people can still develop-to some extent-a conceptual awareness about algorithms and their effects (Cotter \& Reisdorf, 2020; Eslami et al., 2015; Zarouali et al., 2021). In an online media context, conceptual awareness would mean that users know that there is a dynamic system in place that can personalize and customize the information that they see or hear, based on a corpus of data composed of digital traces (Hargittai et al., 2020; Zarouali et al., 2021).

To date, only a limited body of research has focused on people's algorithmic awareness (Hargittai et al., 2020). These studies focus on specific mediated contexts, such as algorithmic curation in social media newsfeeds (Eslami et al., 2015; Rader \& Gray, 2015; Zarouali et al., 2021), online search (Cotter \& Reisdorf, 2020), and news platforms (Powers, 2017). Although the results of these studies are not entirely univocal, we can conclude that the findings so far show that people are characterized by a lack of awareness of algorithmic content curation (e.g., Eslami et al., 2015; Powers, 2017). In addition to this, studies have also shown that there is a strong variation in algorithmic awareness among certain parts of the population (Hargittai et al., 2020; Rader \& Gray, 2015). This has been referred to as the "algorithmic knowledge gap," which might contribute to a new digital divide, and thus merits further investigation (Cotter \& Reisdorf, 2020). Therefore, this study aims to provide a more solid empirical ground by focusing on the prevalence of algorithmic (mis)conceptions, and discussing these findings in the light of digital divides.

The importance of providing more solid empirical insights into algorithmic awareness and algorithmic misconceptions is also important from the perspective of algorithmic accountability and the construction of digital citizenship. Social media users, as digital citizens, have an important role in critically scrutinizing algorithms and the services they are enabling, but also in challenging or resisting algorithms that conflict with users' rights and interests (Hintze et al., 2019). Algorithmic awareness becomes a precondition for algorithmic accountability. Meijer and Grimmelikhuijsen (2021) describe algorithmic accountability as "the justification of the organizational usage of an algorithm and explanations for its outcomes to an accountability forum that can ask questions, pass judgement, and impose consequences" (p. 60). In order to be able to ask the necessary questions and hold controllers of algorithms accountable, users need to possess what Koene et al. (2019) call "algorithmic literacy," along with the ability to act and exercise agency. It is not difficult to see how algorithmic misconceptions and misleading imaginaries inhibit the ability of users to exercise critical citizenship and thereby hold algorithmic power to account. This also explains why so many public policy measures are directed at increasing algorithmic awareness through transparency and media literacy initiatives (see European Commission, 2020a; highlighting the importance of algorithmic awareness to enhancing the ability of individuals to be aware of their rights and know how to act upon them, see Council of Europe, 2020, p. 8). Empirical insights into algorithmic misconceptions, therefore, contribute to both the literature on algorithmic accountability, as well as law and policymaking. 


\subsection{Algorithmic Misconceptions}

When it comes to new technologies, history tells us that their introduction most often goes hand-in-hand with a broad range of projected hopes and fears, which gives rise to various myths and misconceptions (Natale \& Ballatore, 2020). These misconceptions and myths should be seen as dynamic constructs that give meaning and represent an important part of the collective mentality of (a group of) people (Mosco, 2004). Based on a thorough literature review in the area of (machine learning) algorithms, we identified five important misconceptions. This list might not be exhaustive, but it does certainly comprise the major misconceptions highlighted in recent academic work (e.g., de Saint Laurent, 2018; Emmert-Streib et al., 2020; First, 2018; Roffel \& Evans, 2018).

The first major misconception refers to the idea that algorithms are completely independent from human influence. Algorithms are designed by humans to automate certain tasks in a highly optimized way (i.e., being much more efficient than humans) (Gillespie, 2014; Lee, 2018). Importantly, the degree of automation in algorithms can vary: Certain algorithms allow some degree of human involvement, whereas others take fully automated decisions and keep humans completely out of the loop (Diakopoulos, 2019; Parasuraman et al., 2000). In reality, many algorithms do not operate fully independently, but are closely monitored by human beings, they often rely on human-generated input and data and are the result of models and metrics developed by humans (Fry, 2019). That is, they are often used to improve a system's performance, without necessarily reducing human involvement (Shneiderman et al., 2018). In addition, algorithms are constantly being tweaked, tuned, re-written, repaired, or deleted; as such, they are not fully independent technical objects (Kitchin, 2014; Seaver, 2018) and co-evolve in their interactions with humans.

The second misconception is the idea that algorithms are operating neutrally and objectively, and thus, are free of bias. Indeed, algorithms "as such" are unbiased because they are inert and meaningless systems; the bias occurs when algorithms are paired with (humangenerated) databases or models that determine their functioning (Gillespie, 2014). Algorithms can not only display the biases of those who make and operate them, but potentially also the values and (commercial) preferences of the companies that provide them, or the technical infrastructures in which they operate (Ananny \& Crawford, 2018; Gillespie, 2010). So, in reality, all operating algorithms can have some kind of bias (de Saint Laurent, 2018). For instance, subtle human biases (e.g., ideologies, prejudices, and inequalities) can slip into the data inputted, the training of the data, and the algorithmic operation (Amoore \& Piotukh, 2015; Beckett, 2019; van Dijck et al., 2018). On a technical level, research indicated that biases related to data representativeness and sampling can also occur (Eubanks, 2017;
Fry, 2019; Hargittai, 2020). Therefore, algorithmic biases should be seen as reflections of more fundamental societal (and technical) biases (Bucher, 2018).

A third misconception entails that algorithms can replace the high-level critical reasoning and human thought. To illustrate this misconception, take the example of neural networks algorithms. These algorithms can learn to make quicker and more accurate decisions based on experience: The more examples they are exposed to, the more accurate they become (Chesney \& Citron, 2019; Dack, 2019). That is why people came to believe that algorithms mimic the decision-making processes in our human brains. However, as argued by Emmert-Streib et al. (2020), assuming that these algorithmic models perform just like human brains is not plausible nor realistic. In fact, a major downside of algorithms is rooted in their inability to make critical decisions, cope with unanticipated scenarios, make subjective value-based judgments, and display creativity (Diakopoulos, 2019; Shneiderman et al., 2018). Therefore, scholars argue that algorithms cannot (yet) reason in the same way as humans (Roffel \& Evans, 2018).

A fourth misconception is that algorithms can solve every problem in society. In the past decade, many people came to believe that every societal problem or difficulty has a solution based on technology, which has been referred to as "technological solutionism" (Morozov, 2014). This trend toward finding quick technological fixes is combined with an at times somewhat naive trust in the infallibility of technology. The reality is far more nuanced: Algorithms are usually used for solving very specific (rule-based) tasks or problems (Fry, 2019; Roffel \& Evans, 2018) as they excel at executing routine, tedious, and error-prone tasks highly efficiently, tirelessly, and consistently (Diakopoulos, 2019; Shneiderman et al., 2018). Therefore, scholars have cautioned against the idea of considering algorithms as silver bullets that will solve everything (Morozov, 2014; Roffel \& Evans, 2018). Rather, they should be seen as tools that have become very efficient in solving narrow problems.

The fifth misconception is that algorithms will replace human workers in the media sector. A good example would be automation in the newsroom: Some people foresee the elimination of jobs, with human journalists being replaced by algorithms. In reality, algorithms are unlikely to replace journalists, but instead, are often being used to design efficient and effective systems that support workflows and reporting (Beckett, 2019; Diakopoulos, 2019). As with other technological revolutions, it is possible that certain tasks or even professions in the newsroom may become obsolete, but at the same time, the introduction of algorithmic processes also introduces entirely new roles and tasks (Ferrer-Conill $\&$ Tandoc, 2018). Also from the perspective of managerial staff, editors, and journalists, there is no real immediate concern of replaceability in the newsroom; for them, algorithms represent a supplementary (useful) toolkit (Schapals \& Porlezza, 2020). As this illustration shows, 
algorithms should be seen as tools that can support rather than replace human decision-making (Fry, 2019).

\subsection{Research Questions}

Many of the misconceptions discussed above may have (deeply) infiltrated our society. On a societal level, this could lead to some serious concerns, such as a polluted (and myth-based) public debate, but also misjudging the role that humans have, e.g., in the process of spreading misinformation or contributing to algorithmic biases. In addition, algorithmic misconceptions can also seriously undermine the full potential of algorithms in our society (de Saint Laurent, 2018), since governments and other institutions might have a misguided lack of trust (as a result of misconceptions) in the use of algorithmic solutions for societal problems. On the level of the individual user, one of the biggest concerns is that these misconceptions might not be universally distributed in the population, and thus, that they might be overrepresented in certain (more vulnerable) parts of the population, resulting in new forms of digital exclusion. That is, unequal skills and knowledge (including misconceptions) can result in new forms of digital divide, e.g., the "algorithmic divide" (Carmi \& Yates, 2020). In addition, when (certain groups of) people have numerous misconceptions, they might develop a distorted and ill-informed mindset about how algorithms work, which could undermine their ability to make correct and rational judgments about the information that algorithms present to them online and misjudge their own role in the process.

Therefore, this study aims to investigate the prevalence and the main sources of these algorithmic misconceptions in the population. Broadly speaking, when it comes to ICT knowledge and digital literacy skills, many studies have already acknowledged the importance of individual differences by looking at demographic characteristics (e.g., Hargittai, 2010; Schreurs et al., 2017; van Dijk \& van Deursen, 2014). In the context of algorithms, a recent study found a relationship between algorithmic knowledge and socioeconomic background, indicating a worrisome (digital) knowledge inequality (Cotter \& Reisdorf, 2020). Therefore, in this study, we explore the prevalence and differences in algorithmic misconceptions among certain demographic groups (age, gender, and education), as well as investigate whether these demographic variables can predict algorithmic misperceptions. In addition, we also aim to look into the main information sources of algorithmic misperceptions. In particular, knowing the (perceived) sources of misconceptions is essential to be able to refute them effectively (Menz et al., 2021). That is, to overcome the persistence of algorithmic misconception, we must have an idea of the main sources associated with these misconceptions. Therefore, we explore the main information sources that people attribute to their misconceptions and test whether there is a relationship between particular sources and the prevalence of algorithmic mis- conceptions. Based on this, we suggest the following research questions: RQ1) How prevalent are algorithmic misconceptions in the population, and how are they distributed among socio-demographic characteristics (age, gender, and education)?; RQ2) What are the main information sources that people attribute to their algorithmic (mis)conceptions?; and RQ3) Are these demographic characteristics (gender, age, and education) and information sources significant predictors of algorithmic misconceptions (is there a significant association)?

\section{Methods}

\subsection{Sample}

We used data from a larger panel wave study which was distributed among a representative sample of the Dutch population. The larger panel study focused on the societal impact of communication technologies and algorithms. Representativeness was achieved based on age, gender, education, and region. The fieldwork was carried out by a research company. The total sample size was $N=2,106$. To achieve this net sample size, a gross sample of 6,000 people was initially contacted, which means that the overall response rate was $35 \%$. The data collection took place from July 19 to August 9, 2019 (21 days). The respondents had a mean age of 54.18 ( $S D=15.59$ years), and $48 \%$ of them were women. All respondents successfully completing the survey received an incentive (bonus points) from the research company. A demographic overview of the sample is presented in Table 1.

\subsection{Measures}

We measured algorithmic misconceptions by presenting respondents with five true/false statements. To make these statements less abstract, they were preceded by a short explanation about algorithms: "The following questions are about your awareness of the use of algorithms in the media (e.g., algorithms that recommend relevant content to you)." This short introduction was followed by five statements that we discussed above, in the literature review. More precisely, we asked that respondents "indicate whether you believe the following statements about algorithms in the media are true or false," with the following items: (1) Algorithms are completely independent, without human influence; (2) algorithms always operate neutrally and objectively, and thus, are free of bias; (3) algorithms can solve every problem in society; (4) algorithms have the same level of critical reasoning and intelligence as humans; (5) algorithms will replace humans workers in the media sector. All items were misconceptions, i.e., responses needed to be "false" to be correct. Thus, a respondent answering "true" on an item (which is an incorrect answer), is considered as someone holding that specific misconception. We adopted this format, in which all correct responses are "false," from the study of Taylor and Kowalski (2004). In addition, 
Table 1. Socio-demographic characteristics of the representative Dutch sample.

\begin{tabular}{|c|c|c|}
\hline & Percentage (\%) & Frequency $(N)$ \\
\hline \multicolumn{3}{|l|}{ Age categories $\left(M_{\text {age }}=54.18, S D_{\text {age }}=15.59\right)$} \\
\hline $18-34$ years & 14.62 & 308 \\
\hline $35-54$ years & 33.67 & 709 \\
\hline $55+$ years & 51.71 & 1,089 \\
\hline \multicolumn{3}{|l|}{ Gender } \\
\hline Female & 47.96 & 1,010 \\
\hline Male & 52.04 & 1,096 \\
\hline \multicolumn{3}{|l|}{ Education } \\
\hline Low & 30.82 & 649 \\
\hline Moderate & 50.47 & 1,063 \\
\hline High & 18.71 & 394 \\
\hline \multicolumn{3}{|l|}{ Region } \\
\hline North & 11.16 & 235 \\
\hline East & 21.37 & 450 \\
\hline South & 24.17 & 509 \\
\hline West & 28.40 & 598 \\
\hline Three large cities (Amsterdam, Rotterdam, and The Hague) & 14.91 & 314 \\
\hline
\end{tabular}

we also created an aggregated measure for algorithmic misconceptions (for the purpose of multivariate analyses). To do this, all incorrect answers were coded with 1 , and summed to compute an index with scores ranging from 0 to 5 . A higher score on this index means that person holds more algorithmic misconceptions $(M=2.31$, $S D=1.75)$.

To investigate the information sources of algorithmic misconceptions, we used a similar approach as Menz et al. (2021). We asked respondents to indicate what sources contributed to their acquisition of algorithmic information. They were given multiple answer options: (1) own experiences, (2) media (offline and online), (3) school, (4) friends and/or family, (5) no information source, (6) other. We manually checked all responses of the "other," and many of the responses could easily be categorized in the five other options, which we did. Therefore, this category was not included in the analyses.

In terms of socio-demographic information, we measured respondents' age, gender, education level, and geographical region. Age was measured on a continuous level (for the multivariate analyses) and was also recoded into a categorical variable consisting of three age groups. Gender was measured based on two response choices: female and male. Education was measured based on a detailed list of seven categories (tailored to the Dutch education system). This categorization can be re-coded into three education levels: low (no education or primary education), moderate (secondary education), and high (post-secondary and higher education). The variable region was based on Nielsen's regional division of the Netherlands, which is the gold standard in market research. We provide the (sample) descriptives of this variable, but we do not include them in the statistical analyses.

\section{Results}

\subsection{Prevalence and Distribution of Algorithmic Misconceptions (RQ1)}

Table 2 presents the general prevalence of all five algorithmic misconceptions among all respondents (first row), as well as a more narrowed overview of the prevalence in specific demographic groups. The numbers in the table refer to the percentage of people that gave an incorrect answer on a misconception item (see measures), which means the proportion of respondents holding that misconception. Looking at the first row, i.e., the general prevalence numbers, we see that the first, second, fourth, and fifth misperception are supported by more than half of the respondents. The proportion related to the third misperception is slightly lower (43.64\%). In particular, misconception five, i.e., that algorithms will replace human workers, is the most widespread among the respondents (63.96\%). In terms of age groups, Table 1 illustrates that age is significantly associated with the prevalence of all algorithmic misconceptions (see $x^{2}$ tests), except for misconception five. The Z-tests (indicated by means of superscripts) provide a more detailed overview, specifying which proportions differ from each other. Based on these tests, we see that older age groups have more algorithmic misconceptions than younger respondents. Gender was also found to be consistently associated with the prevalence of all misconceptions. More precisely, a higher proportion of women held algorithmic misconceptions than men. Finally, education level was also significantly associated with the extent to which people hold all five algorithmic misconceptions. That is, lower-educated respondents were more likely to have 
Table 2. Percentage of people giving an incorrect answer on the misconception items.

\begin{tabular}{|c|c|c|c|c|c|}
\hline & MC1 & MC2 & MC3 & MC4 & MC5 \\
\hline Total sample (\%) & 53.66 & 52.85 & 43.64 & 54.23 & 63.96 \\
\hline \multicolumn{6}{|l|}{ Age categories (\%) } \\
\hline $18-34$ years & $44.81^{a}$ & $42.21^{a}$ & $39.61^{a}$ & $46.43^{a}$ & $60.06^{a}$ \\
\hline $35-54$ years & $49.51^{a}$ & $43.86^{\mathrm{a}}$ & $37.52^{\mathrm{a}}$ & $48.52^{a}$ & $63.05^{a}$ \\
\hline $55+$ years & $58.86^{b}$ & $61.71^{\mathrm{b}}$ & $48.76^{\mathrm{b}}$ & $60.15^{b}$ & $65.66^{a}$ \\
\hline$x^{2}$-test & $26.48^{* * *}$ & $71.64^{* * *}$ & $24.45^{* * *}$ & $32.23^{* * *}$ & $3.64(n s)$ \\
\hline \multicolumn{6}{|l|}{ Gender (\%) } \\
\hline Male & $50.27^{a}$ & $45.89^{a}$ & $35.31^{\mathrm{a}}$ & $49.18^{a}$ & $60.04^{a}$ \\
\hline Female & $57.33^{b}$ & $60.40^{b}$ & $52.67^{b}$ & $59.70^{b}$ & $68.22^{b}$ \\
\hline$x^{2}$-test & $10.16^{* *}$ & $44.36 * * *$ & $64.43^{* * *}$ & $23.45^{* * *}$ & $15.26 * * *$ \\
\hline \multicolumn{6}{|l|}{ Education (\%) } \\
\hline Low & $63.02^{a}$ & $67.64^{\mathrm{a}}$ & $58.86^{\mathrm{a}}$ & $65.02^{a}$ & $70.72^{a}$ \\
\hline Moderate & $51.36^{b}$ & $51.83^{b}$ & $42.62^{b}$ & $53.25^{b}$ & $63.78^{b}$ \\
\hline High & $44.42^{c}$ & $31.22^{c}$ & $21.32^{c}$ & $39.09^{c}$ & $53.30^{c}$ \\
\hline$x^{2}$-test & $38.66 * * *$ & $131.42 * * *$ & $141.39 * * *$ & $67.28 * * *$ & $32.32 * * *$ \\
\hline
\end{tabular}

Notes: For each variable, proportions in the same column with different superscripts $\left({ }^{a},{ }^{b}, c\right)$ differ significantly at least at $p<0.05$ (z-test). $* * * p<0.001, * * p<0.01, * p<0.05$. MC1: Algorithms are completely independent, without human influence. MC2: Algorithms always operate neutrally and objectively, and thus, are free of bias. MC3: Algorithms can solve every problem in society. MC4: Algorithms have the same level of critical reasoning and intelligence as humans. MC5: Algorithms will replace humans workers in the media sector.

algorithmic misconceptions compared to moderately and higher-educated respondents.

\subsection{Information Sources of Algorithmic Misconceptions (RQ2)}

Table 3 gives a summary of the main information sources of respondents holding algorithmic misconceptions. In this table, we used the algorithmic misconception measure, the index ranging from 0 to 5 , indicating the number of misconceptions held (with 5 meaning that people hold all five misconceptions; and 0, none). Based on this table, we conclude that respondents with more misconceptions tend to rely less on their own experience, media, and school as sources of information. Conversely, they more commonly rely on friends and/or family as information sources, and are particularly likely to have no information source at all (up to $74.48 \%$ ). These results suggest that one's own experiences, media, and school are associated with having fewer algorithmic misconcep- tions, whereas having friends/family as sources or having no information source at all are associated with having more algorithmic misperceptions.

\subsection{Predictors of Algorithmic Misconceptions (RQ3)}

Multiple regression analysis was performed to explore which variables predict algorithmic misconceptions among respondents. The regression model is presented in Table 4. Age was found to be a significant predic tor of algorithmic misconceptions: The older people get, the more algorithmic misperceptions they have $(\beta=0.09, p<0.001)$. Gender was revealed as a positive predictor of misconceptions, with women have significantly more algorithmic misperceptions than men $(\beta=0.11, p<0.001)$. For education, the regression analysis revealed that respondents with moderate $(\beta=-0.08$, $p<0.01)$ and high education $(\beta=-0.15, p<0.001)$ levels have significantly fewer algorithmic misconceptions than respondents with a low education level. Altogether,

Table 3. Information sources in function of people's algorithmic misconception index score.

\begin{tabular}{lrrrrrr}
\hline & \multicolumn{5}{c}{ Algorithmic misconception index score } \\
\cline { 2 - 6 } & 0 & 1 & 2 & 3 & 4 & 5 \\
\hline Own experiences (\%) & $55.30^{\mathrm{a}}$ & $56.80^{\mathrm{a}}$ & $45.61^{\mathrm{b}}$ & $43.77^{\mathrm{b}}$ & $28.09^{\mathrm{c}}$ & $10.48^{\mathrm{d}}$ \\
Media (\%) & $51.89^{\mathrm{a}}$ & $55.20^{\mathrm{a}}$ & $55.37^{\mathrm{a}}$ & $45.45^{\mathrm{b}}$ & $32.34^{\mathrm{c}}$ & $12.38^{\mathrm{d}}$ \\
School (\%) & $9.09^{\mathrm{a}}$ & $9.87^{\mathrm{a}}$ & $8.78^{\mathrm{a}}$ & $6.06^{\mathrm{a}, \mathrm{b}}$ & $2.55^{\mathrm{b}}$ & $1.52^{\mathrm{b}}$ \\
Friends and/or family (\%) & $10.29^{\mathrm{a}}$ & $16.77^{\mathrm{b}}$ & $18.30^{\mathrm{b}}$ & $22.44^{\mathrm{b}}$ & $21.21^{\mathrm{b}}$ & $23.73^{\mathrm{b}}$ \\
No information source (\%) & $20.45^{\mathrm{a}}$ & $16.53^{\mathrm{a}}$ & $20.73^{\mathrm{a}}$ & $27.61^{\mathrm{b}}$ & $43.83^{\mathrm{c}}$ & $74.48^{\mathrm{d}}$ \\
\hline
\end{tabular}

Notes: Proportions in the same row with different superscripts $\left({ }^{a},{ }^{b}, c, d\right)$ differ significantly at least at $p<0.05$ (z-test). 
Table 4. The predictors of algorithmic misconceptions.

\begin{tabular}{|c|c|c|c|c|c|c|}
\hline & & B & SE & $\beta$ & t-value & Sig. \\
\hline Constant & & 1.77 & 0.21 & & 8.42 & $* * *$ \\
\hline \multicolumn{7}{|l|}{ Block 1: Demographics } \\
\hline Age & & 0.01 & 0.01 & 0.09 & 4.60 & $* * *$ \\
\hline Gender & & 0.37 & 0.07 & 0.11 & 5.48 & $* * *$ \\
\hline Moderate education & & -0.26 & 0.08 & -0.08 & -3.34 & $* *$ \\
\hline High education & & -0.66 & 0.10 & -0.15 & -6.41 & $* * *$ \\
\hline$R^{2}(\%)$ & 0.110 & & & & & $* * *$ \\
\hline \multicolumn{7}{|c|}{ Block 2: Information sources } \\
\hline Own experiences & & -0.44 & 0.09 & -0.12 & -5.06 & $* * *$ \\
\hline Media & & -0.32 & 0.09 & -0.09 & -3.59 & $* * *$ \\
\hline School & & -0.12 & 0.14 & -0.02 & -0.83 & $n s$ \\
\hline Friends and/or family & & 0.18 & 0.09 & 0.04 & 1.97 & $*$ \\
\hline No information source & & 0.91 & 0.11 & 0.25 & 8.20 & $* * *$ \\
\hline Incremental $R^{2}$ & 0.135 & & & & & $* * *$ \\
\hline Total $R^{2}(\%)$ & 0.245 & & & & & $* * *$ \\
\hline
\end{tabular}

these demographic variables explained $11 \%$ of the variance. In the second block, we included the information sources. On the one hand, we see that a person's own experience $(\beta=-0.12, p<0.001)$ and media $(\beta=-0.09$, $p<0.001)$ are both sources that are negatively associated with having algorithmic misconceptions. On the other, friends/family $(\beta=0.04, p<0.05)$ and no information source $(\beta=0.25, p<0.001)$ were positively linked to algorithmic misconceptions. Information from school was not significantly linked to algorithmic misconceptions $(\beta=-0.02, n s)$.

\section{Discussion}

This study showed that misconceptions about algorithms in the media are highly prevalent among the general population in the Netherlands (see Table 2). This prevalence is significantly more pronounced among very specific socio-demographic groups. Results showed that age, education, and gender were significant predictors of algorithmic misperceptions. More precisely, we found that erroneous representations about media algorithms are more common among (1) older people (vs. younger people), (2) lower-educated people (vs. higher-educated), and (3) women (vs. men). In addition, this study also explored the information sources that might contribute to these algorithmic misperceptions. It was found that people who have no information sources about algorithms, and those who rely on their friends and family for such information, were more likely to have algorithmic misconceptions. On the other hand, media, school, and people's own experience were found to be sources associated with having fewer algorithmic misconceptions, suggesting that these three are important sources to convey correct and accurate information about algorithms to the general public. All in all, these results tend to suggest that there is a clear variation in algorithmic misconceptions in society (with a higher prevalence among certain vulnerable parts of the population), which might contribute to new digital divides or inequalities (Cotter \& Reisdorf, 2020).

These findings have important contributions to the literature of algorithmic awareness, and the digital divide. As mentioned earlier, the literature on algorithmic awareness is characterized by: 1) a limited body of research; 2) findings that are not entirely conclusive; 3) studies that-almost-exclusively focused on algorithms in specific mediated contexts (e.g., social media algorithms, news algorithms, search algorithms). The contribution of this study is that it focused on people's (mis)conceptions about algorithms on a more general level (without context-specificity) and that it presents insights that might indicate a significant lack of algorithmic awareness among the general population (particularly among certain vulnerable demographic groups). This point then brings us to the second important contribution, i.e., to the digital divide literature. The current findings raise the issue of whether algorithms are expanding digital divides, rather than closing them. It is important that people all have equal skills and knowledge to benefit from algorithmic systems (or at least, have equal opportunities to develop such skills and knowledge); if not, this may create what has been called "algorithmic divides" (Carmi \& Yates, 2020). This divide manifests itself in parts of the population having a clear idea on how to benefit most of algorithmic technologies (e.g., young, high-educated individuals), whereas other parts of the population, including more vulnerable groups, might be excluded from the advantages of these technologies (e.g., older, low-educated individuals) and 
fail to understand the role of algorithms in the media or the role that humans can play in algorithmic processes. As this study made clear differences in misconception visible, we hope that our findings raise awareness of algorithmic misconception as a factor that can contribute to digital exclusion and divides.

Finally, the findings from our study also entail some important contribution to both the literature and the practice of algorithmic accountability. According to recent findings, the Netherlands supposedly belongs to the leading group of EU countries when it comes to digital literacy (highest proportion of residents skilled in using tech; Eurostat, 2020; "The Netherlands ranks," 2020). It is therefore disturbing to see that even in a country with a relatively high level of digital literacy a rather significant part of the population holds algorithmic misconceptions. Recently, the European Commission set out a path to boosting the investment in, and widespread implementation of Al and algorithms (European Commission, 2020b). Europe is on its way to becoming an algorithmic society-a society shaped by the interplay of humans and the coding and processing of information through algorithms, and that increasingly depends on data-driven processes and decision-making systems. The main questions then are: (1) Will citizens be able to ask the right critical questions about the role and functioning of digital technology?, (2) does the population possess the necessary level of literacy to benefit from these systems?, and (3) are users sufficiently prepared to recognized and protect themselves from possible negative consequences of these technologies? A society in which a significant share of users hold (serious) misconceptions about the potential and workings of algorithms is hardly able to engage critically with algorithmic solutions. Such a society might not be prepared to decide where and how (not) to use algorithms and may be unable to understand their own role in algorithmic processes or to compel those who wield algorithmic power to respect their fundamental rights and public values. The lack of critical digital citizenship, again, can become a potential source of societal problems, such as the spread of misinformation and deep fakes, data-driven manipulation and re-enforcing stereotypes, and inequalities and discrimination (Eubanks, 2017; Mohamed et al., 2020). Our study raises a number of critical follow-up questions, for example regarding the literature on algorithmic transparency and accountability. Transparency is often discussed as a tool to overcome the information asymmetries between users, governments, and corporations, but is it also the role of transparency to correct misconceptions? Or would this require different interventions? To what extent can transparency enhancing measures even contribute to the creation of misconceptions? Can consent to data processing be considered "informed" in the sense of the GDPR if it based on misconceptions? What additional regulatory, policy, and organizational safeguards are needed to empower users to be able to hold algorithmic power to account? But also, where are the limits to "accountability by user empowerment" if users are not able to make fully informed decisions because these are based on misconceptions?

Our study also underlines the urgency of digital literacy education programs and more attention to the role of algorithms in the media. In such literacy initiatives, it is important to balance the need to equip citizens with protective strategies to face the harmful consequences of online algorithms, but at the same time, also to focus on empowering them with a nuanced appreciation of what algorithms are-and what they are notand the ways in which algorithms may benefit individuals and society (Hobbs, 2020). These initiatives can be implemented within education programs at school or offered via mediated channels-two sources that we found to be particularly influential in debunking algorithmic misconceptions. It is also important to pay particular attention to vulnerable groups that are much harder to reach via schools and media, such as older age groups and lower-educated people. For these groups, a more tailored approach might be needed. Related to this, the results of this study seem to align with the argument that we should be wary of labelling young people as "digital natives" (Kirschner \& De Bruyckere, 2017; Kirschner \& van Merriënboer, 2013). Although the younger respondents in this study had lower levels of algorithmic misconceptions compared to older age groups, we can still conclude that these misconceptions are present among a considerable share of young adults. Based on these insights, labelling them as digital natives might obscure their need for support in developing the necessary skills to correctly understand algorithmic processes in the media. Therefore, future discussions about educational policy and practice should not be embedded in a mindset that considers young people-by default-as wellversed in algorithmic technology (i.e., digital natives), but rather from the perspective that further education and training is needed to teach them about the uses and consequences of algorithms.

Finally, we also want to address some limitations that could inspire future research. First, this research is based on a very exploratory and descriptive analysis of algorithmic misconceptions. We, therefore, encourage scholars to examine this topic in more depth, with the current study serving as a starting point. There are still many questions that remain unanswered, such as: What are the root causes of these misconceptions? Is the prevalence of algorithmic misconceptions in other countries comparable? Are these misconceptions caused by high levels of trust in the capabilities of algorithms (technological solutionism), or by a lack of critical thinking? What are the consequences of these misconceptions for the willingness to use or trust digital technology? A second limitation relates to the misconceptions selected in this study. They were chosen because of their importance and prominence in the literature. But, our list of misconceptions is by no means exhaustive, meaning that there can be still other algorithmic misconceptions in our 
society. Therefore, we encourage scholars to capture the full breadth of misperceptions. This will help to get a better image of algorithmic awareness of citizens. A third limitation relates to the unpredictability of future algorithmic developments. That is, the algorithmic misconceptions discussed in this study might not necessarily be misconceptions in the future. For instance: Algorithms are not-significantly-replacing media workers at this point, but it would not be unreasonable to expect that in some far-distant future, humans might be replaced by efficient algorithms in certain media sectors. So, whether these misconceptions will eventually stand the test of time, remains to be seen and so keeping track of how these misconceptions develop through time would provide an interesting avenue for future research. Finally, this study did not measure variables related to familiarity with technology and digital media. It would therefore be interesting to investigate how these misconceptions relate to technological savviness and digital literacy and to explore how important these factors are in forming algorithmic misconceptions.

\section{Acknowledgments}

This work was funded by the Research Priority Area ICDS from the University of Amsterdam.

\section{Conflict of Interests}

The authors declare no conflict of interests.

\section{References}

Amoore, L., \& Piotukh, V. (2015). Life beyond big data: Governing with little analytics. Economy and Society, 44(3), 341-366. https://doi.org/10.1080/03085147. 2015.1043793

Ananny, M., \& Crawford, K. (2018). Seeing without knowing: Limitations of the transparency ideal and its application to algorithmic accountability. New Media \& Society, 20(3), 973-989. https://doi.org/10.1177/ 1461444816676645

Beckett, C. (2019, November 18). New powers, new responsibilities: A global survey of journalism and artificial intelligence. Polis. https://blogs.Ise.ac.uk/ polis/2019/11/18/new-powers-new-responsibilities

Beer, D. (2017). The social power of algorithms. Information, Communication \& Society, 20(1), 1-13. https:// doi.org/10.1080/1369118X.2016.1216147

Bucher, T. (2018). If...then: Algorithmic power and politics. Oxford University Press.

Carmi, E., \& Yates, S. J. (2020). What do digital inclusion and data literacy mean today? Internet Policy Review, 9(2). https://doi.org/10.14763/2020.2.1474

Chesney, B., \& Citron, D. (2019). Deep fakes: A looming challenge for privacy, democracy, and national security. California Law Review, 107(6), 1753-1820.

Cotter, K., \& Reisdorf, B. C. (2020). Algorithmic knowledge gaps: A new dimension of (digital) inequality. International Journal of Communication, 14, 745-765.

Council of Europe. (2020). Recommendation CM/ $\operatorname{Rec}(2020) 1$ of the committee of ministers to member states on the human rights impacts of algorithmic systems. https://search.coe.int/cm/pages/ result_details.aspx?objectid=09000016809e 1154

Dack, S. (2019, March 20). Deep fakes, fake news, and what comes next. The Henry M. Jackson School of International Studies. https://jsis.washington.edu/ news/deep-fakes-fake-news-and-what-comes-next

de Saint Laurent, C. (2018). In defence of machine learning: Debunking the myths of artificial intelligence. Europe's Journal of Psychology, 14(4), 734-747. https://doi.org/10.5964/ejop.v14i4.1823

Diakopoulos, N. (2019). Automating the news: How algorithms are rewriting the media. Harvard University Press.

Elish, M. C., \& boyd, d. (2018). Situating methods in the magic of big data and Al. Communication Monographs, 85(1), 57-80. https://doi.org/10.1080/ 03637751.2017.1375130

Emmert-Streib, F., Yli-Harja, O., \& Dehmer, M. (2020). Artificial intelligence: A clarification of misconceptions, myths and desired status. Frontiers in Artificial Intelligence, 3, 1-7.

Eslami, M., Rickman, A., Vaccaro, K., Aleyasen, A., Vuong, A., Karahalios, K., Hamilton, K., \& Sandvig, C. (2015). "I always assumed that I wasn't really that close to [her]": Reasoning about invisible algorithms in news feeds. Proceedings of the 33rd Annual ACM Conference on Human Factors in Computing Systems-CHI'15 (pp. 153-162). ACM. https://doi. org/10.1145/2702123.2702556

Eubanks, V. (2017). Automating inequality: How hightech tools profile, police, and punish the poor (1st ed.). St. Martin's Press.

European Commission. (2020a). Communication from the Commission to the European Parliament, the Council, the European Economic and Social Committee and the Committee of the Regions on The European democracy action plan (COM(2020) 790). European Commission. https://eur-lex.europa.eu/legalcontent/EN/TXT/?uri=COM:2020:790:FIN

European Commission. (2020b). Shaping Europe's digital future. Publications Office of the European Union. https://ec.europa.eu/info/sites/default/files/ communication-shaping-europes-digital-futurefeb2020_en_4.pdf

Eurostat. (2020). Individuals' level of digital skills [Data set]. https://appsso.eurostat.ec.europa.eu/nui/ show.do?dataset=isoc_sk_dskl_i\&lang=en

Ferrer-Conill, R., \& Tandoc, E. C. (2018). The audienceoriented editor: Making sense of the audience in the newsroom. Digital Journalism, 6(4), 436-453. https://doi.org/10.1080/21670811.2018.1440972

First, D. (2018). Will big data algorithms dismantle the foundations of liberalism? AI \& SOCIETY, 33(4), 
545-556. https://doi.org/10.1007/s00146-0170733-4

Frank, M., Roehrig, P., \& Pring, B. (2017). What to do when machines do everything: How to get ahead in a world of Al, algorithms, bots, and big data. Wiley.

Fry, H. (2019). Hello world: How to be human in the age of the machine. Transworld Publishers.

Gillespie, T. (2010). The politics of "platforms." New Media \& Society, 12(3), 347-364. https://doi.org/ $10.1177 / 1461444809342738$

Gillespie, T. (2014). The relevance of algorithms. In T. Gillespie, P. J. Boczkowski, \& K. A. Foot (Eds.), Media technologies (pp. 167-194). MIT Press. https://doi. org/10.7551/mitpress/9780262525374.003.0009

Hargittai, E. (2010). Digital na(t)ives? Variation in internet skills and uses among members of the "net generation." Sociological Inquiry, 80(1), 92-113. https:// doi.org/10.1111/j.1475-682X.2009.00317.x

Hargittai, E. (2020). Potential biases in big data: Omitted voices on social media. Social Science Computer Review, 38(1), 10-24. https://doi.org/10.1177/ 0894439318788322

Hargittai, E., Gruber, J., Djukaric, T., Fuchs, J., \& Brombach, L. (2020). Black box measures? How to study people's algorithm skills. Information, Communication \& Society, 23(5), 764-775. https://doi.org/ 10.1080/1369118X.2020.1713846

Hintze, A., Dencik, L., \& Wahl-Jorgensen, K. (2019). Digital citizenship in a datafied society. Polity.

Hobbs, R. (2020). Propaganda in an age of algorithmic personalization: Expanding literacy research and practice. Reading Research Quarterly, 55(3), 521-533. https://doi.org/10.1002/rrq.301

Kirschner, P. A., \& De Bruyckere, P. (2017). The myths of the digital native and the multitasker. Teaching and Teacher Education, 67, 135-142. https://doi.org/ 10.1016/j.tate.2017.06.001

Kirschner, P. A., \& van Merriënboer, J. J. G. (2013). Do learners really know best? Urban legends in education. Educational Psychologist, 48(3), 169-183. https://doi.org/10.1080/00461520.2013.804395

Kitchin, R. (2014). The data revolution: Big data, open data, data infrastructures \& their consequences. SAGE.

Kitchin, R. (2017). Thinking critically about and researching algorithms. Information, Communication \& Society, 20(1), 14-29. https://doi.org/10.1080/ 1369118X.2016.1154087

Koene, A., Clifton, C., Hatada, Y., Webb, H., Patel, M., Machaod, C., LaViolette, J., Richardson, R., \& Reisman, D. (2019). A governance framework for algorithmic accountability and transparency. European Parliament. https://www.europarl.europa.eu/ RegData/etudes/STUD/2019/624262/EPRS STU(2019)624262_EN.pdf

Lee, M. K. (2018). Understanding perception of algorithmic decisions: Fairness, trust, and emotion in response to algorithmic management. Big Data \&
Society, 5(1). https://doi.org/10.1177/2053951718 756684

Meijer, A., \& Grimmelikhuijsen, S. (2021). Responsible and accountable algorithmization: How to generate citizen trust in governmental usage of algorithms. In M. Schuilenburg \& R. Peters (Eds.), The algorithmic society: Technology, power, and knowledge (pp. 53-66). Routledge.

Menz, C., Spinath, B., \& Seifried, E. (2021). Where do pre-service teachers' educational psychological misconceptions come from? The roles of anecdotal versus scientific evidence. Zeitschrift Für Pädagogische Psychologie, 35(2/3), 1-14. https://doi.org/10.1024/ 1010-0652/a000299

Mohamed, S., Png, M.-T., \& Isaac, W. (2020). Decolonial Al: Decolonial theory as sociotechnical foresight in artificial intelligence. Philosophy \& Technology, 33(4), 659-684. https://doi.org/10.1007/ s13347-020-00405-8

Morozov, E. (2014). To save everything, click here: Technology, solutionism, and the urge to fix problems that don't exist. Penguin Books.

Mosco, V. (2004). The digital sublime: Myth, power, and cyberspace. MIT Press.

Natale, S., \& Ballatore, A. (2020). Imagining the thinking machine: Technological myths and the rise of artificial intelligence. Convergence: The International Journal of Research into New Media Technologies, 26(1), 3-18. https://doi.org/10.1177/1354856517715164

Parasuraman, R., Sheridan, T. B., \& Wickens, C. D. (2000). A model for types and levels of human interaction with automation. IEEE Transactions on Systems, Man, and Cybernetics-Part A: Systems and Humans, 30(3), 286-297. https://doi.org/10.1109/ 3468.844354

Pasquale, F. (2015). The black box society: The secret algorithms that control money and information. Harvard University Press.

Powers, E. (2017). My news feed is filtered? Digital Journalism, 5(10), 1315-1335. https://doi.org/10.1080/ 21670811.2017.1286943

Rader, E., \& Gray, R. (2015). Understanding user beliefs about algorithmic curation in the Facebook news feed. Proceedings of the 33rd Annual ACM Conference on Human Factors in Computing Systems-CHI'15 (pp. 173-182). ACM. https://doi. org/10.1145/2702123.2702174

Ricci, F. (2015). Recommender systems handbook. Springer.

Roffel, S., \& Evans, I. (2018, July 16). The biggest misconceptions about Al: The experts' view. Elsevier. https://www.elsevier.com/connect/the-biggestmisconceptions-about-ai-the-experts-view

Schapals, A. K., \& Porlezza, C. (2020). Assistance or resistance? Evaluating the intersection of automated journalism and journalistic role conceptions. Media and Communication, 8(3), 16-26. https://doi.org/ 10.17645/mac.v8i3.3054 
Schreurs, K., Quan-Haase, A., \& Martin, K. (2017). Problematizing the digital literacy paradox in the context of older adults' ICT use: Aging, media discourse, and self-determination. Canadian Journal of Communication, 42(2). https://doi.org/10.22230/cjc. 2017v42n2a3130

Seaver, N. (2018). What should an anthropology of algorithms do? Cultural Anthropology, 33(3), 375-385. https://doi.org/10.14506/ca33.3.04

Shneiderman, B., Plaisant, C., Cohen, M., Jacobs, S., \& Elmqvist, N. (2018). Designing the user interface: Strategies for effective human-computer interaction. Pearson.

Taylor, A. K., \& Kowalski, P. (2004). Naïve psychological science: The prevalence, strength, and sources of misconceptions. The Psychological Record, 54(1), 15-25.
https://doi.org/10.1007/BF03395459

The Netherlands ranks among the EU top in digital skills. (2020, February 2). CBS. https://www.cbs.nl/en-gb/ news/2020/07/the-netherlands-ranks-among-theeu-top-in-digital-skills

van Dijck, J., Poell, T., \& de Waal, M. (2018). The platform society. Oxford University Press.

van Dijk, J. A. G. M., \& van Deursen, A. J. A. M. (2014). Digital skills: Unlocking the information society. Palgrave Macmillan. https://doi.org/10.1057/978113743703

Zarouali, B., Boerman, S. C., \& de Vreese, C. H. (2021). Is this recommended by an algorithm? The development and validation of the algorithmic media content awareness scale (AMCA-scale). Telematics and Informatics, 62, Article 101607. https://doi.org/10.1016/ j.tele.2021.101607

\section{About the Authors}
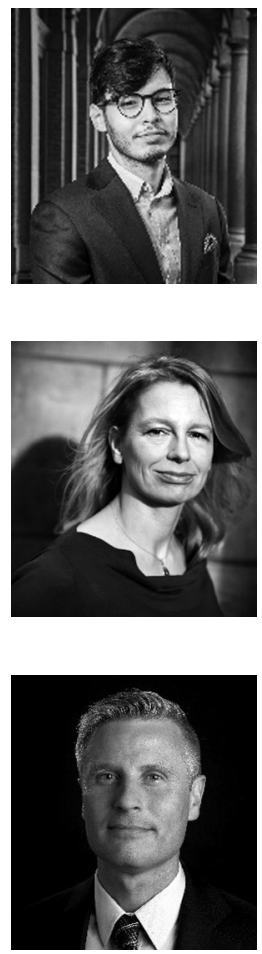

Brahim Zarouali is an assistant professor in persuasion and new media technologies at the Amsterdam School of Communication Research (ASCoR) of the University of Amsterdam. His interests can be situated at the intersection of persuasive communication and new media technologies (e.g., recommendation algorithms, chatbots, virtual assistants, etc.), with a specific focus on how these technologies shape individuals' views, attitudes, and behaviours. He usually focuses on the broader implications of these technologies on our society as a whole.

Natali Helberger is a distinguished university professor of law and digital technology, with a special focus on Al, and affiliated with the Institute for Information Law (IVIR) of the University of Amsterdam. Her research focus is on how digitization, algorithms, and Al are transforming the media and the implications for public values, diversity in the media landscape, and the media's democratic role.

Claes $\mathbf{H}$. de Vreese is a distinguished faculty professor of artificial intelligence, data, and democracy and professor of political communication at the Amsterdam School of Communication Research (ASCoR) and University of Amsterdam (UvA). His research interests include the role of data and artificial intelligence in democratic processes, microtargeting, comparative journalism research, the effects of news, public opinion, and European integration. 